\title{
THE ECONOMIC SUBSTANTIATION FOR PRODUCTION OF GASTRIC TEAS AS PROMISING MEDICINES FOR TREATING GASTRO-INTESTINAL TRACT DISEASES
}

\author{
O.A.Kyslychenko, A.I.Fedosov, O.V.Dorovskyy, R.V.Sahaidak-Nikitiuk \\ National University of Pharmacy
}

Key words: gastric tea; gastro-intestinal tract; treatment of diseases; economic substantiation; production

The dynamics of gastro-intestinal tract diseases has been investigated in the article. The methods of treatment of these diseases have been studied. The marketing analysis of phytoteas has been carried out. The composition of phytoteas presented at the Ukrainian pharmaceutical market has been studied. The composition of phytoteas used for gastro-intestinal tract disorders has been developed and suggested. The approaches of the economic substantiation of reasonability for production of the phytoteas suggested are given. The prices for these phytoteas have been substantiated, and their prime cost has been calculated. The economic efficacy of the research on development of the phytotea composition has been determined.

Diseases and disorders of the gastro-intestinal tract (GIT) represent a very important part of clinical medicine research due to their high prevalence among the patients, complexity of pathogenesis, the lack of effectiveness of the existing treatment methods and the relapsing course of these diseases. Gastroenterology achievements, search, directed synthesis and the study of the new effective medicines show that the problem of GIT diseases treatment is of great importance and interest at the moment $[8,14]$.

Medicinal plants (MP) and teas on their basis occupy a special place in the complex therapy of GIT diseases. This is due to the fact that phytotherapy is well tolerated by patients and almost does not show side-effects. MP teas can be taken simultaneously with other treatment methods suggested by modern medicine that allows patients to recover quicker and more effective. In some difficult cases the use of phytotherapy in parallel with pharmacotherapy increases not only the effectiveness, but also the safety of treatment. Such effect can be explained by the membrane stabilizing action and elimination of metabolites, toxins and cellular decomposition products with the blood circulation from the human organism. In some cases if there are no symptoms of acute inflammation, phytotherapy can be chosen as main therapy. It is also used for the relapsing course of GIT diseases prophylaxis in case of their chronic course.

The use of MP teas is especially of a great interest at the moment while the anti-terroristic operation at the Eastern territories of Ukraine is taking place. The main part of surgeries requires rehabilitation. The long period of rehabilitation requires application of medicines with a soft action and few side effects. The irregular and unbalanced diet (especially during military campaigns) can become the cause for exacerbation of chronic diseases [15].

Medicines of the plant origin have been traditionally used in Ukraine for centuries. Nowadays, the use of phyto- therapy is very urgent not only because of its proven effectiveness and minimal side effects (it becomes extremely important during the long-term treatment), but also because of the low price of these medicines.

These days there is a crisis in Ukraine in providing the population with medicines with the low price and good quality. The patients' incomes decrease while prices on drugs increase. According to this fact, the volume of drug consumption in Ukraine is determined not only by the level of morbidity of the population and the needs of the healthcare system in medicines, but also by the purchase ability of patients [2].

During the last years there is a trend of GIT diseases increase. For example, the number of patients with this nosology in 2013 was 2764 persons per 100 thousand of the population, i.e. 1254856 people [2].

The results of the questioning conducted indicate the trend of the future consumption of phytoteas and teas by the patients will be approximately $55 \%$ despite the low level of solvency. In the expert questionary 352 patients took part. The number of patients who will use phytoteas for treating the digestion system disorders (the dosage form is teas in filter bags or cartons weighing $100 \mathrm{~g}$ ) will equal approximately 690 thousand people $[2,16]$.

\section{Materials and Methods}

When conducting the research the legal, economic, marketing and pharmaceutical methods were used $[6,9,14]$.

\section{Results and Discussion}

The main step of providing the population with drugs is substantiation of the production necessity of certain medicines. The economic substantiation for production expediency of phytoteas was conducted in three steps:

- the first step - marketing research of the pharmaceutical market;

- the second step - calculation of the economic efficacy of manufacturing of the phytotea prime cost; 
- the third step - the economic substantiation of the scientific work efficacy [5-17].

Nowadays there are a lot of phytoteas for GIT treatment at the pharmaceutical market of Ukraine. The main manufacturers presented at the market are: "AIM" Ltd, "Kluchi zdoroviya" research-and-production pharmaceutical company, "Novaya formatsiya" Ltd, "Regionalniy Tsentr Fitochayov" Ltd and others. Marketing research is presented in Table.
The research conducted shows the necessity of developing and introducing phytoteas for treating GIT diseases into manufacture at the domestic pharmaceutical enterprises despite their wide assortment at the market. On the one hand, this is due to the great amount of plants in the composition of a phytotea that can cause allergy. On the other hand, it limits the segment of consumers because of the prevalence of allergic diseases among patients. The third reason is high prices for the existing medicines.

Table

The comparative characteristics of phytoteas

\begin{tabular}{|c|c|c|c|c|}
\hline $\begin{array}{l}\text { The name } \\
\text { of a phytotea }\end{array}$ & Manufacturer & Composition & Indications for use & $\begin{array}{l}\text { Price, } \\
\text { UAH }\end{array}$ \\
\hline 1 & 2 & 3 & 4 & 5 \\
\hline $\begin{array}{l}\text { TianDe } \\
\text { Phytotea } \\
\text { "Gastric tea } \\
\text { with mar- } \\
\text { shmallow" }\end{array}$ & TianDe & $\begin{array}{ll}\text { 1. } & \text { Bergenia leaves (Bergenia crassifolia) } \\
\text { 2. } & \text { Marshmallow root (Althaea officinalis) } \\
\text { 3. } & \text { Angelica root (Angelica sinensis) } \\
\text { 4. } & \text { Flax seed (Linum usitatissimum) } \\
\text { 5. } & \text { Chamomile flowers (Matricaria } \\
\text { 6. } & \text { chamomilla) } \\
\text { 7. } & \text { Licorice root (Glycyrrhiza glabra) } \\
\text { 8. } & \text { Chicory root (Cichorium intybus) }\end{array}$ & $\begin{array}{l}\text { It restores the gastric } \\
\text { functional activity and } \\
\text { protects against organic } \\
\text { diseases; improves the } \\
\text { gastric mucosa state and } \\
\text { normalizes digestion }\end{array}$ & 56.00 \\
\hline $\begin{array}{l}\text { "Gastro- } \\
\text { intestinal" } \\
\text { phytotea }\end{array}$ & $\begin{array}{l}\text { Manufacturer } \\
\text { "Krymskiy sbor" }\end{array}$ & $\begin{array}{ll}\text { 1. } & \text { Nettle (Urtica diö̈ca) } \\
\text { 2. } & \text { Rubus leaves (Rubus subg Rubus) } \\
\text { 3. } & \text { Peppermint (Mentha piperita) } \\
\text { 4. } & \text { Catnip (Nepeta cataria) } \\
\text { 5. } & \text { Hyssop (Hyssopus officinalis) } \\
\text { 6. } & \text { Lime (Tilia cordata) } \\
\text { 7. } & \text { German chamomile (Matricaria } \\
& \text { chamomilla) }\end{array}$ & $\begin{array}{l}\text { It is a prophylactic } \\
\text { medicine against GIT } \\
\text { diseases }\end{array}$ & 39.00 \\
\hline $\begin{array}{l}\text { Gastro- } \\
\text { intestinal } \\
\text { phytotea } \\
\text { "Gastrophyt" }\end{array}$ & $\begin{array}{l}\text { AIM Ltd, } \\
\text { Research-and- } \\
\text { production } \\
\text { pharmaceutical } \\
\text { company }\end{array}$ & $\begin{array}{l}\text { 1. } \text { Calamus rhizome (Acorus calamus) } \\
\text { 2. Marshmallow root (Althaea officinalis) } \\
\text { 3. Helichrysum flowers (Helichrysum } \\
\text { arenarium) } \\
\text { 4. Black elder flowers (Sambucus nigra) } \\
\text { 5. St. John's wort herb (Hypericum } \\
\text { 6erforatum) } \\
\text { 6. Calendula flowers (Calendula officinalis) } \\
\text { 7. Nettle leaves (Urtica dioïca) } \\
\text { 8. Peppermint leaves (Mentha Piperita) } \\
\text { 9. Wormwood herb (Artemisia absinthium) } \\
\text { 10. German chamomile flowers (Matricaria } \\
\text { 11. Pagoda tree fruit (Sophora japonica) } \\
\text { 12. Licorice root (Glycyrrhiza glabra) } \\
\text { 13. Yarrow herb (Achillea Millefolium) } \\
\text { 14. Sage leaves (Salvia officinalis) } \\
\text { 15. Cinnamon rose fruit (Rosa cinnamomea) }\end{array}$ & $\begin{array}{l}\text { It has spasmolytic } \\
\text { properties; stimulates the } \\
\text { reparative processes in } \\
\text { the gastric and duodenal } \\
\text { mucosa; regulates the GIT } \\
\text { functioning; normalizes } \\
\text { the intestinal motility; } \\
\text { has the choleretic and } \\
\text { carminative activity }\end{array}$ & 29.00 \\
\hline $\begin{array}{l}\text { "Gastro- } \\
\text { intestinal" } \\
\text { phytotea }\end{array}$ & "Megan" Ltd & $\begin{array}{ll}\text { 1. } & \text { Calamus (Acorus calamus) } \\
\text { 2. } & \text { Marshmallow (Althaea officinalis) } \\
\text { 3. } & \text { Elecampane (Inula helenium) } \\
\text { 4. } & \text { St. John's wort herb (Hypericum } \\
& \text { perforatum) } \\
\text { 5. } & \text { Calendula (Calendula officinalis) } \\
\text { 6. Peppermint (Mentha piperita) } \\
\text { 7. German chamomile flowers (Matricaria } \\
\text { 8hamomilla) } \\
\text { 8. Yarrow (Achillea Millefolium) } \\
\text { 9. } & \text { Wild thyme herb (Thymus serpyllum) }\end{array}$ & $\begin{array}{l}\text { It has the spasmolytic, } \\
\text { antibacterial and anti- } \\
\text { inflammatory action; } \\
\text { improves the GIT } \\
\text { functioning; has tonic } \\
\text { properties }\end{array}$ & 25.00 \\
\hline
\end{tabular}


Table continuation

\begin{tabular}{|c|c|c|c|c|}
\hline 1 & 2 & 3 & 4 & 5 \\
\hline $\begin{array}{l}\text { Phytotea } \\
\text { "Kurilskiy } \\
\text { chai" }\end{array}$ & $\begin{array}{l}\text { "Sibirskoye } \\
\text { zdorovye" } \\
\text { corporation }\end{array}$ & $\begin{array}{l}\text { 1. Bupleurum herb (Bupleurum aureum) } \\
\text { 2. Plantain leaf (Plantago major) } \\
\text { 3. Cinnamon rose fruit (Rosa cinnamomea) } \\
\text { 4. German chamomile flowers (Matricaria } \\
\text { chamomilla) }\end{array}$ & $\begin{array}{l}\text { It has the regenerating } \\
\text { effect on the digestive } \\
\text { system; exhibits } \\
\text { the choleretic and } \\
\text { hepatoprotective effects; } \\
\text { normalizes the intestinal } \\
\text { microflora; eliminates fats } \\
\text { and cholesterol }\end{array}$ & 35.00 \\
\hline $\begin{array}{l}\text { "Phyto } \\
\text { gastro- } \\
\text { intestinal" } \\
\text { phytotea } \\
\text { No. } 7\end{array}$ & $\begin{array}{l}\text { TM "Klyuchi } \\
\text { zdorovya" }\end{array}$ & $\begin{array}{ll}\text { 1. } & \text { Calamus rhizome (Acorus calamus) } \\
\text { 2. } & \text { Marshmallow root (Althaea officinalis) } \\
\text { 3. } & \text { Elecampane root (Inula helenium) } \\
\text { 4. } & \text { Pot majoram herb (Origanum vulgare) } \\
\text { 5. } & \text { St. John's wort herb (Hypericum } \\
& \text { perforatum) } \\
\text { 6. } & \text { Yarrow herb (Achillea Millefolium) } \\
\text { 7. } & \text { Wild thyme herb (Thymus serpyllum) } \\
\text { 8. } & \text { Peppermint leaves (Mentha piperita) } \\
\text { 9. } & \text { German chamomile flowers (Matricaria } \\
& \text { chamomilla) } \\
\text { 10. } & \text { Calendula flowers (Calendula officinalis) }\end{array}$ & $\begin{array}{l}\text { It normalizes the GIT } \\
\text { functions }\end{array}$ & 13.95 \\
\hline $\begin{array}{l}\text { Gastro- } \\
\text { intestinal } \\
\text { phytotea No. } \\
5 \text { "Doctor+" }\end{array}$ & $\begin{array}{l}\text { Krivosheyev K.G. } \\
\text { Private company }\end{array}$ & $\begin{array}{l}\text { 1. } \text { Cinnamon rose fruit (Rosa cinnamomea) } \\
\text { 2. } \\
\text { 3. }\end{array}$ & $\begin{array}{l}\text { It has the spasmolytic, } \\
\text { antibacterial and anti- } \\
\text { inflammatory action; } \\
\text { improves the GIT } \\
\text { functioning; has tonic } \\
\text { properties }\end{array}$ & 12.55 \\
\hline $\begin{array}{l}\text { Gastro- } \\
\text { intestinal } \\
\text { phytotea } \\
\text { No. } 5\end{array}$ & $\begin{array}{l}\text { "Regionalny } \\
\text { centr } \\
\text { phytochayov" } \\
\text { Ltd }\end{array}$ & $\begin{array}{l}\text { 1. Cinnamon rose fruit (Rosa cinnamomea) } \\
\text { 2. St. John's wort herb (Hypericum } \\
\text { perforatum) } \\
\text { 3. Wild thyme herb (Thymus serpyllum) } \\
\text { 4. Nettle leaves (Urtica diö̈ca) } \\
\text { 5. Sage leaves (Salvia officinalis) } \\
\text { 6. Peppermint leaves (Mentha piperita) } \\
\text { 7. Lime flowers (Tilia cordata) } \\
\text { 8. Plantain leaf (Plantago major) } \\
\text { 9. Coltsfoot leaves (Tussilago farfara) } \\
\text { 10. Calamus rhizome (Acorus calamus) } \\
\text { 11. German chamomile flowers (Matricaria } \\
\text { 12. } \text { Yarrow grass (Achillea millefolium) } \\
\text { 13. Common knotgrass herb (Polygonum } \\
\text { aviculare) } \\
\text { 14. Chaga mushroom (Inonotus obliquus) } \\
\text { 15. Stevia leaves (Stevia rebaudiana) }\end{array}$ & $\begin{array}{l}\text { It has the spasmolytic, } \\
\text { antibacterial and ant- } \\
\text { inflammatory action; } \\
\text { improves the GIT activity; } \\
\text { has tonic properties }\end{array}$ & 35.95 \\
\hline
\end{tabular}


Table continuation

\begin{tabular}{|c|c|c|c|c|}
\hline 1 & 2 & 3 & 4 & 5 \\
\hline $\begin{array}{l}\text { "Gastro- } \\
\text { intestinal» } \\
\text { phytotea of } \\
\text { Carpathian } \\
\text { species }\end{array}$ & $\begin{array}{l}\text { "Karpatskiy } \\
\text { sbor" Ltd }\end{array}$ & $\begin{array}{ll}\text { 1. } & \text { Common knotgrass (Polygonum aviculare) } \\
\text { 2. } & \text { Oats (Avena sativa) } \\
\text { 3. } & \text { Fireweed (Chamerion angustifolium) } \\
\text { 4. } & \text { German chamomile flowers (Matricaria } \\
\text { chamomilla) } \\
\text { 5. Peppermint leaves (Mentha piperita) } \\
\text { 6. St. John's wort herb (Hypericum } \\
\text { perforatum) } \\
\text { 7. } \\
\text { Rubus leaves (Rubus subg. Rubus) } \\
\end{array}$ & $\begin{array}{l}\text { It is a prophylactic } \\
\text { medicine against GIT } \\
\text { diseases }\end{array}$ & 30.00 \\
\hline $\begin{array}{l}\text { "Gastro- } \\
\text { intestinal with } \\
\text { Iceland moss" } \\
\text { phytotea }\end{array}$ & $\begin{array}{l}\text { "Naturalis" } \\
\text { company }\end{array}$ & $\begin{array}{ll}\text { 1. } & \text { Iceland moss (Cetraria islandica) } \\
\text { 2. } & \text { Marshmallow root (Althaea officinalis) } \\
\text { 3. Licorice root (Glycyrrhiza glabra) } \\
\text { 4. German chamomile flowers (Matricaria } \\
\text { 5hamomilla) } \\
\text { 5. Dill fruit (Anethum graveolens) } \\
\text { 6. Fennel fruit (Foeniculum vulgare) } \\
\text { 7. Agrimony herb (Agrimonia eupatoria) } \\
\text { 8. St. John's wort herb (Hypericum } \\
\text { perforatum) } \\
\text { 9. Wild thyme herb (Thymus serpyllum) } \\
\text { 10. Common bilberry leaves and shoots } \\
\text { (Vaccinium myrtillus) } \\
\text { 11. Coriander leaves and fruit (Coriandrum } \\
\text { sativum) } \\
\text { 12. Oats straw (Avena sativa) } \\
\text { 13. Common knotgrass herb (Polygonum } \\
\text { aviculare) } \\
\text { 14. Calendula flowers (Calendula officinalis) } \\
\text { 15. Tormentil root (Potentilla erecta) } \\
\text { 16. Peppermint leaves (Mentha piperita) } \\
\text { 17. Nettle herb (Urtica dioïca) } \\
\text { 18. Common bean (Phasoleous vulgaris) } \\
\text { 19. Marsh cudweed (Gnaphalium uliginosum) } \\
\text { 20. Motherwort herb (Leonurus cardiaca) } \\
\text { 21. Oak bark cut (Quercus robur) } \\
\text { 22. Nettle leaves (Urtica diö̈ca) } \\
\text { 23. Sage leaves (Salvia officinalis) }\end{array}$ & $\begin{array}{l}\text { It relieves spasms of the } \\
\text { stomach and intestines; } \\
\text { relieves digestion; helps } \\
\text { to restore the normal } \\
\text { pancreas functioning; } \\
\text { improves functioning of } \\
\text { the liver and intestine; } \\
\text { improves metabolism; } \\
\text { relieves flatulence, belch } \\
\text { and heartburn }\end{array}$ & 25.00 \\
\hline $\begin{array}{l}\text { "Sila } \\
\text { rossiyskih } \\
\text { trav No. 5" } \\
\text { phytotea }\end{array}$ & "Vitachai" Ltd & 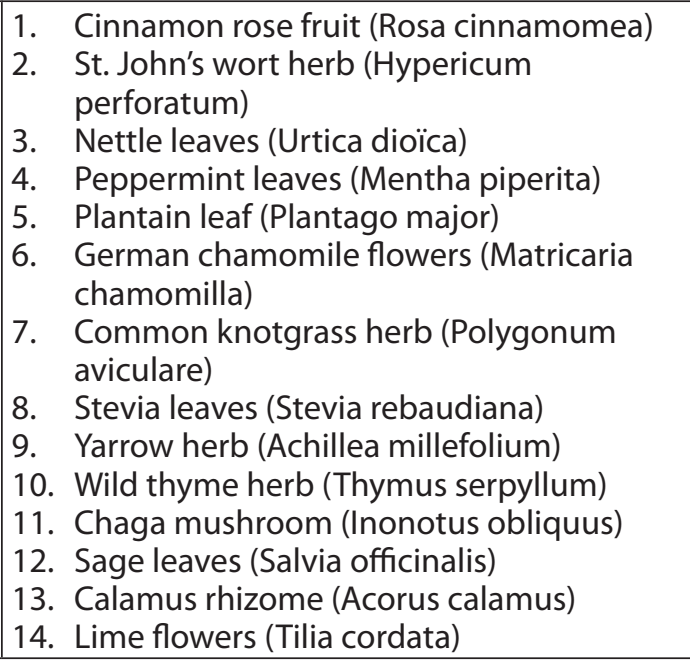 & $\begin{array}{l}\text { It has the spasmolytic, } \\
\text { antibacterial and anti- } \\
\text { inflammatory action; } \\
\text { improves the GIT } \\
\text { functioning; has tonic } \\
\text { properties }\end{array}$ & 15.00 \\
\hline
\end{tabular}


Table continuation

\begin{tabular}{|c|c|c|c|c|}
\hline 1 & 2 & 3 & 4 & 5 \\
\hline $\begin{array}{l}\text { Gastric tea } \\
\text { prepared } \\
\text { according to } \\
\text { the ancient } \\
\text { Monastery } \\
\text { recipe }\end{array}$ & Belarus & $\begin{array}{ll}\text { 1. } & \text { Calendula flowers (Calendula officinalis) } \\
\text { 2. } & \text { Flaxseed seed } \\
\text { 3. } & \text { Cinnamon rose fruit (Rosa cinnamomea) } \\
\text { 4. } & \text { St. John's wort herb (Hypericum } \\
& \text { perforatum) } \\
\text { 5. } & \text { Marsh cudweed (Gnaphalium uliginosum) } \\
\text { 6. } & \text { eppermint leaves (Mentha piperita) } \\
\text { 7. } & \text { Wormwood herb (Artemisia absinthium) } \\
\text { 8. } & \text { Equisetum herb (Equisetum arvense) } \\
\text { 9. } & \text { Yarrow flowers (Achillea millefolium) }\end{array}$ & $\begin{array}{l}\text { The tea is used for } \\
\text { treatment of any GIT } \\
\text { diseases: } \\
\text { - chronic gastritis; } \\
\text { - gastric and duodenal } \\
\text { ulcers; } \\
\text { - colitis of any etiology; } \\
\text { - bacterial and fungal } \\
\text { intestinal infections; } \\
\text { - intoxications of different } \\
\text { etiology - poisonings } \\
\text { by poi-sons, drugs or } \\
\text { alcohol; } \\
\text { - defecation problems } \\
\text { (constipation and } \\
\text { diarrhea); } \\
\text { - enzymatic insuffisiency; } \\
\text { - flatulence; } \\
\text { - intestinal dysbacteiosis, etc }\end{array}$ & 230.00 \\
\hline
\end{tabular}

That is why the second step is substantiation of the cost of the phytotea proposed. The prime cost calculation for production of 1000 packs of the gastric phytotea for conditions of the pharmaceutical manufacture " $\mathrm{A}$ " (confidential information) includes the following items:

1. The main plant raw material.

2. Auxiliary materials.

3. Salary, including taxes.

4. Manufacturing expenses.

5. Administrative expenses.

6. Sales costs (expenses).

7. Other operating expenses.

Full cost is $6721 \mathrm{UAH}$.

Thus, based on the calculations and marketing research conducted it has been determined that the optimal price of a phytotea per pack is $8.50 \mathrm{UAH}$. In such a case, the profit of the manufacture obtained, including the number of patients when they use only 1 pack of the phytotea equals $613755 \mathrm{UAH}$.

The third step is the economic substantiation of the scientific work efficacy. Expenses on the scientific work refer to production costs. They are one-time costs, which include such items:

- materials;
- $\quad$ energy costs;

- $\quad$ special equipment for research;

- main salary;

- additional salary;

- taxes on salary;

- business trips expenses;

- $\quad$ payment for utility services;

- expenses on work carried out by other organizations;

- other direct expenses;

- other overheads.

On the basis of the standard method [1, 3, 4] it has been determined that the research cost equals 145928.90 UAH, and the price of the scientific and technical production will be $185928.90 \mathrm{UAH}$. The profitability index of investments for assessing a scientific invention in the field of pharmacy is 0.27 .

\section{CONCLUSIONS}

According to the research conducted the importance of development and production of the gastro-intestinal phytotea has been proven, its acceptable price has been determined, and the indicators of the scientific work efficacy have been calculated.

\section{REFERENCES}

1. Агарков А.П., Голов Р.С., Голиков А.М. Организация производства: интегрированное учебное пособие. - М.: Дашков и Ко, 2012. - 329 с.

2. Посилкіна О.В., Сагайдак-Нікітюк Р.В., Деренська Я.М. Економіка, планування та організачія хімікофармацевтичного виробництва: методичні рекомендачії до виконання курсової роботи та економічної частини дипломного проекту у вигляді наукової роботи для студентів спеціальності 7.12020103 «Технологія фармацевтичних препаратів». - Х.: Вид-во НФаУ, 2015. - 53 с.

3. Рязанова В., Люшина Э. Организачия и планирование производства: пособие. - М.: Академия, 2010. 272 c.

4. Статистичний щорічник Украӥни за 2013 рік / За ред. О.Г.Осауленка. - К.: Державна служба статистики України, 2014. - 534 с. 
5. Carrots and sticks. Sustainability reporting policies worldwide - today's best practice, tomorrow's trends [Электронный ресурс]. - Режим доступа: https://www.globalreporting.org/resourcelibrary/Carrots-andSticks.pdf

6. EU measures for small and medium-sized enterprises (SMEs) [Электронный pecypc]. - Режсим доступа: http://www.russell-cooke.co.uk/client Upload/ document/file/EU \%20measures\% 20for \%20SMEs\%20-\%20 June\%202011.pdf.

7. Graves C.W. // ECLET Publishing, 2005. - 578 p.

8. GRI: Empowering Sustainable Decisions Our five-year focus 2015-2020 [Электронный ресурс]. - Режсим доступа: https://www.globalreporting.org/information/news-and-press-center/Documents/GRI-Five-year-focus-2015.pdf

9. Kutshera U., Niklas K.J. // Naturwissenschaften. - 2004. - Vol. 91, №6. - P. 255-276.

10. Mizhnarodnyi standart bukhhalters 'koho obliku 2 "Zapasy" [Электронный ресурс]. - Режим доступа: http://zakon.nau.ua/doc/?uid=1014.7729.0.

11. Mizhnarodnyi standart bukhhalters'koho obliku 41 "Sil's'ke hospodarstvo" [Электронный ресурс]. - Режим docmyna: http://www.minfin.gov.ua/control/publish/article/main?art_id=92410\&cat_id=92408.

12. Mizhnarodnyi standart finansovoi zvitnosti 13 "Otsinka spravedlyvoi vartosti” [Электронный ресурс]. Pежим достуnа: http://www.minfin.gov.ua/file/link/364291/file/IFRS13.pdf.

13. Peter N. Instrumentos de Regulación Monetaria [Электронный ресурс]. - Режим достуnа: https://www2. bc.edu/peter-ireland/ec261/chapter17a.pdf

14. Stachowiak Z. // Theory and practice of security mechanism. - 2012. - №1. - P. 14-18.

15. Sugonyako D.O., Bazilinska O.Y. // Actual problems of modern economy development: Collection of scientific articles. - Thorpe-Bowker ${ }^{\circledR}$, Melbourne, Australia, 2015. - P. 145-149.

16. Sustainability and Reporting Trends in 2025: Preparing for the Future [Электронный ресурс]. - Режсим доступа: https://www.globalreporting. org/resourcelibrary/Sustainability-and-Reporting-Trends-in-2025-1.pdf.

17. The official site of Global Reporting Initiative [Электронный ресурс]. - Режим достуnа: www.globalreporting.org.

ЕКОНОМІЧНЕ ОБґРУНТУВАННЯ ВИРОБНИЦТВА ШЛУНКОВИХ ЗБОРІВ ЯК ПЕРСПЕКТИВНИХ ЗАСОБІВ ЛІКУВАННЯ ЗАХВОРЮВАНЬ ШЛУНКОВО-КИШКОВОГО TРАКТУ

О.А.Кисличенко, А.І.Федосов, О.В.Доровський, Р.В.Сагайдак-Нікітюк

Ключові слова: шлунковий збір; шлунково-кишковий тракт; лікування захворювань; економічне обгрунтування; виробництво

Досліджено динаміку захворюваності шлунково-кишкового тракту, розглянуті методи лікування цього захворювання. Проведено маркетинговий аналіз ринку фіточаїв. Досліджено склад фріточаїв, представлених на ринку України. Запропоновано склад фріточаїв для лікування кишково-шлункових захворювань. Наведені підходи економічного обгрунтування доцільності виробництва запропонованих фріточаїв. Обірунтовані ціни на ці чаї та розраховано їх собівартість. Визначена економічна ефективність наукової роботи з розробки складу фріточаїв.

\section{ЭКОНОМИЧЕСКОЕ ОБОСНОВАНИЕ ПРОИЗВОДСТВА ЖЕЛУДОЧНЫХ СБОРОВ КАК ПЕРСПЕКТИВНЫХ СРЕДСТВ ЛЕЧЕНИЯ ЗАБОЛЕВАНИЙ ЖЕЛУДОЧНО-КИШЕЧНОГО TPAKTA}

\section{А.А.Кисличенко, А.И.Федосов, А.В.Доровской, Р.В.Сагайдак-Никитюк}

Ключевые слова: желудочный сбор, желудочно-кишечный тракт, лечение заболеваний, экономическое обоснование, производство

Исследована динамика заболеваемости желудочно-кишечного тракта, рассмотрены методы лечения этого заболевания. Проведен маркетинговый анализ рынка фриточаев. Исследован состав фриточаев, представленных на рынке Украины. Предложен состав фриточаев, используемых для лечения желудочно-кишечных заболеваний. Приведены подходы экономического обоснования целесообразности производства предложенных фриточаев. Обоснованы цены на эти чаи и рассчитана их себестоимость. Определена экономическая эфффективность научной работы по разработке состава фичточаев. 